\title{
Statistical Modeling of Low SNR Magnetic Resonance Images in Wavelet Domain Using Laplacian Prior and Two-Sided Rayleigh Noise for Visual Quality Improvement
}

\begin{abstract}
H. Rabbani
Biomedical Engineering Department, Medical Image and Signal Processing Research Center, Isfahan University of Medical Sciences, Isfahan, Iran, h_rabbani@med.mui.ac.ir

In this paper we introduce a new wavelet-based image denoising algorithm using maximum a posteriori (MAP) criterion. For this reason we propose Laplace distribution with local variance for clean image and two-sided Rayleigh model for noise in wavelet domain. The local Laplace probability density function (pdf) is able to simultaneously model the heavy-tailed nature of marginal distribution and intrascale dependency between spatial adjacent coefficients. Using local Laplace prior and two-sided Rayleigh noise, we derive a new shrinkage function for image denoising in the wavelet domain. We propose our new spatially adaptive wavelet-based image denoising algorithm for several low signal-to-noise ratio (SNR) magnetic resonance (MR) images and compare the results with other methods. The simulation results show that this algorithm is able to truly improve the visual quality of noisy MR images with very low computational cost. In case the input MR image is blurred, a blind deconvolution (BD) algorithm is necessary for visual quality improvement. Since BD techniques are usually sensitive to noise, in this paper we also apply a BD algorithm to an appropriate subband in the wavelet domain to eliminate the effect of noise in the BD procedure and to further improve visual quality.
\end{abstract}

Keywords: Wavelet transform, magnetic resonance images, blind deconvolution, maximum a posteriori estimator

\section{INTRODUCTION}

$\mathrm{M}$ AGNETIC RESONANCE IMAGING (MRI) is a powerful diagnostic technique. Usually in this imaging device, noise contaminates the data during image acquisition and may degrade the human interpretation and post processing tasks.

Usually, MRI noise arises from various sources, including physiological processes, stochastic variation, eddy currents, RF fields generated from electronic circuitry, variations of the magnetic field which arise from susceptibility differences between tissues (off-resonance effects), body motion, etc [1-2].

The impact of noise is increased due to some limitations in acquisition time (e.g., patient comfort, system throughput and real time application), resulting in images with low visual quality and signal-to-noise ratio (SNR). Early methods of denoising, such as spatial filtering, blur the edges while reducing the noise.

In the last two decades, there has been a fair amount of researchers working on wavelet-based image denoising [39]. The problem of wavelet based image denoising is expressed as an estimation problem in a Bayesian framework. Using maximum a posteriori (MAP) estimator, the solution requires a prior knowledge about the distribution of noise and wavelet coefficients.

Since the electronic noise in the real and imaginary parts of the raw MR data is assumed to be independently Gaussian, usually the stochastic noise of magnitude of MR images is modeled by a Rician distribution [10]. However, noise of low and high SNR data can be approximated by Rayleigh probability density function (pdf) and Gaussian pdf [10], respectively. This paper is focused on low SNR MR images, and so we propose a Rayleigh pdf for noise.
Note that these models are appropriate for stochastic noise and in order to remove the non-stochastic noise components, such as \%off-resonance artifacts and rigid body motion, other techniques, such as deblurring methods, are required.

The compression property of wavelets states that the marginal pdf of wavelets in each subband is heavy-tailed [6], [11]. To model this property, various pdfs such as Gaussian, Laplace, generalized Gaussian and mixture pdfs have been proposed [8-9], [11]. Another property of wavelets is clustering. This property states that if a particular wavelet coefficient is large/small, then spatial adjacent coefficients are very likely large/small too [8]. Usually spatially-adaptive algorithms that use local pdfs are able to model this property [5], [9]. In order to take into account compression and clustering properties, we propose Laplacian pdf with local variance for clean wavelet coefficients.

In some cases, the initial MR images are blurred while we may need to focus on image details. To solve this problem, we need to apply a blind deconvolution (BD) algorithm to restore the unblurred image. However, many BD algorithms [12-15] are sensitive to noise and fail in the presence of (high level) noise. In this paper we use a BD algorithm in the low-pass subband of wavelet transform (that approximately excludes the noise) and obtain an image with highlighted details.

This paper, which is an extension of our previous conference paper [16], is organized as follows. In Section 2 the theoretical base of denoising with MAP estimator based on local Laplacian prior and two-sided Rayleigh noise is introduced. In Section 3 we use our wavelet-based denoising algorithm for enhancement of several low SNR MR images. In Section 4 we use a BD technique in the wavelet domain for further improvement of blurred data. Finally the 
concluding remarks are given in Section 5.

Note that in our previous works [9], [17-18] we employ mixture pdf to capture the heavy-tailed nature of wavelet coefficients. In this paper we use a simple non-mixture model (Laplacian pdf) that decreases the computational complexity. Although this model is simpler, using a more complex model only will be useful when we have enough data for accurate estimation (of model parameters). Since we use a local window around each coefficient to capture the intrascale dependency (local pdf), which is the main important dependency between coefficients, we must choose an appropriate distribution for data within the window. For instance, for a $3 \times 3$ window we must fit an appropriate distribution to data using just 9 observations, and if we use a model with many parameters, our estimations may not be reliable. In this base, our simulations confirm that using a simple local Laplacian prior in many cases can compete with the mixture model. In addition, the proposed distribution for noise in [17] is Gaussian. In this paper, we estimate the Rician noise, which is the accurate model for noise in MRI, with Rayleigh noise (for high level noise) and obtain another closed form for thresholding. In spite of previous works, the main purpose of this paper is deblurring, not denoising, and the MAP-based denoising algorithm is used in high-pass subbands (for noisy cases) before deblurring in the wavelet domain [9], [16-18].

\section{PRoposed SPATIALly-ADAPTIVE WAVELET-BASED DENOISING METHOD}

The goal of this section is to estimate the clean signal from noisy observations using the MAP criterion. Let $\mathrm{y}(\mathrm{k})=\mathrm{w}(\mathrm{k})+\mathrm{n}(\mathrm{k}), \mathrm{w}(\mathrm{k})$ and $\mathrm{n}(\mathrm{k})$ represent the low SNR MR image, clean image and noise in wavelet domain, respectively.

The MAP estimator $\hat{w}(k)$ is defined by:

$$
\hat{w}(k)=\underset{w(k)}{\arg \max } p_{w(k) \mid y(k)}(w(k) \mid y(k))
$$

Using Bayes' rule we would have:

$$
\hat{w}(k)=\underset{w(k)}{\arg \max }\left[p_{y(k) \mid w(k)}(y(k) \mid w(k)) p_{w(k)}(w(k))\right]
$$

From $\mathrm{y}(\mathrm{k})=\mathrm{w}(\mathrm{k})+\mathrm{n}(\mathrm{k})$ we have:

$$
p_{y(k) \mid w(k)}(y(k) \mid w(k))=p_{n}(y(k)-w(k)) \text {. }
$$

Therefore, we can write:

$$
\hat{w}(k)=\underset{w(k)}{\arg \max }\left[\log \left(p_{n}(y(k)-w(k))\right)+\log \left(p_{w(k)}(w(k))\right)\right]
$$

and we can obtain the MAP estimate of $w(k)$ by setting the derivative to zero with respect to $\mathrm{w}(\mathrm{k})$ that gives the following equation to solve for $\mathrm{w}(\mathrm{k})$ :

$$
\frac{d \log \left(p_{n(k)}(y(k)-w(k))\right)}{d w(k)}+\frac{d \log \left(p_{w(k)}(w(k))\right)}{d w(k)}=0
$$

In this paper we use a Laplacian pdf with local variance for clean data in the wavelet domain:

$$
p_{w(k)}(w(k))=\frac{1}{\sigma(k) \sqrt{2}} \exp \left(-\sqrt{2} \frac{|w(k)|}{\sigma(k)}\right)
$$

Therefore, we have:

$$
\log \left(p_{w(k)}(w(k))\right)=-\log (\sigma(k) \sqrt{2})-\sqrt{2}|w(k)| / \sigma(k) \quad \text { that }
$$

leads to the following equation:

$$
\frac{d \log \left(p_{n(k)}(y(k)-w(k))\right)}{d w(k)}-\frac{\sqrt{2}}{\sigma(k)} \operatorname{sign}(w(k))=0
$$

It has been shown that the noise of low SNR images is modeled by a two-sided Rayleigh pdf [10]:

$$
p_{n(k)}(n(k))=\frac{1}{2 \alpha^{2}} \cdot \exp \left(-\frac{n^{2}(k)}{2 \alpha^{2}}\right)
$$

where $\alpha=\sigma_{n} / \sqrt{2}$ and $\sigma_{n}$ indicates the noise variance. Substituting (7) to (6) we obtain:

$$
\frac{-1}{y(k)-w(k)}+\frac{y(k)-w(k)}{\alpha^{2}}-\frac{\sqrt{2}}{\sigma(k)} \operatorname{sign}(w(k))=0
$$

By solving the above equation, the MAP estimator comes out to be:

$\hat{w}(k)=\operatorname{sign}(y(k)) \cdot \max \left(0,|y(k)|-\frac{\alpha^{2}}{\sigma(k) \sqrt{2}}+\sqrt{\left.\frac{\alpha^{4}}{2 \sigma^{2}(k)}+\alpha^{2}\right)}\right.$

Fig.1 shows the nonlocal version of the shrinkage function (9) for various parameters. It is clear that for a constant $\sigma$, the data will be more shrunk for larger $\alpha$. In contrast, for a constant $\alpha$, the data will be more shrunk for smaller $\sigma$.

To implement the obtained shrinkage function (9) we need to estimate the parameters $\alpha$ and $\sigma(k)$. The noise variance $\sigma_{n}^{2}$ is estimated using a robust median estimator for the finest scale of noisy wavelet coefficients [3]. So, $\alpha=\sigma_{n} / \sqrt{2}$ is obtained as follows:

$$
\alpha=\frac{\operatorname{median}\left(\left|y_{i}\right|\right)}{0.6745 \sqrt{2}}
$$

The clean image variance $\sigma^{2}(k)$ is estimated by averaging over a squared window $\mathrm{N}(\mathrm{k})$ centered at $\mathrm{k}[5]$ :

$$
\hat{\sigma}^{2}(k)=\max \left(\frac{1}{<N(k)>} \sum_{j \in \mathrm{N}(k)} y^{2}(j) / M-\sigma_{n}^{2}, 0\right)
$$


where $\langle N(k)\rangle$ is the number of coefficients in $\mathrm{N}(\mathrm{k})$.

The proposed denoising algorithm using the above thresholding function is concluded in Table 1.

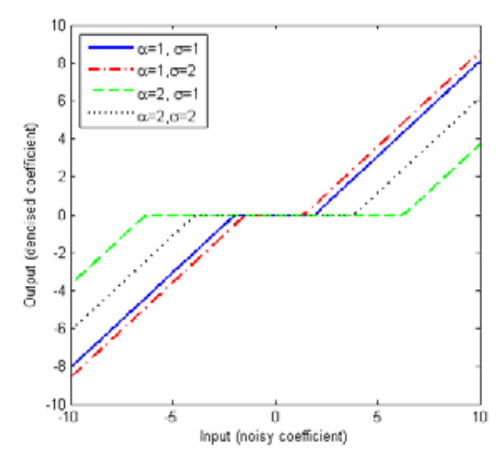

Fig.1. Shrinkage function (9) for various parameters.

Table 1. Proposed denoising algorithm in this paper

\begin{tabular}{|l|l|}
\hline Step 1 & Take the wavelet transform of the low SNR observation. \\
\hline Step 2 & Estimate $\alpha$ from noisy coefficients using $(10)$. \\
\hline Step 3 & For each wavelet coefficient, estimate the variance $\sigma^{2}(k)$ using (11). \\
\hline Step 4 & Substitute the obtained parameters in previous two steps in (9). \\
\hline Step 5 & Take the inverse wavelet transform of the reconstructed image. \\
\hline
\end{tabular}

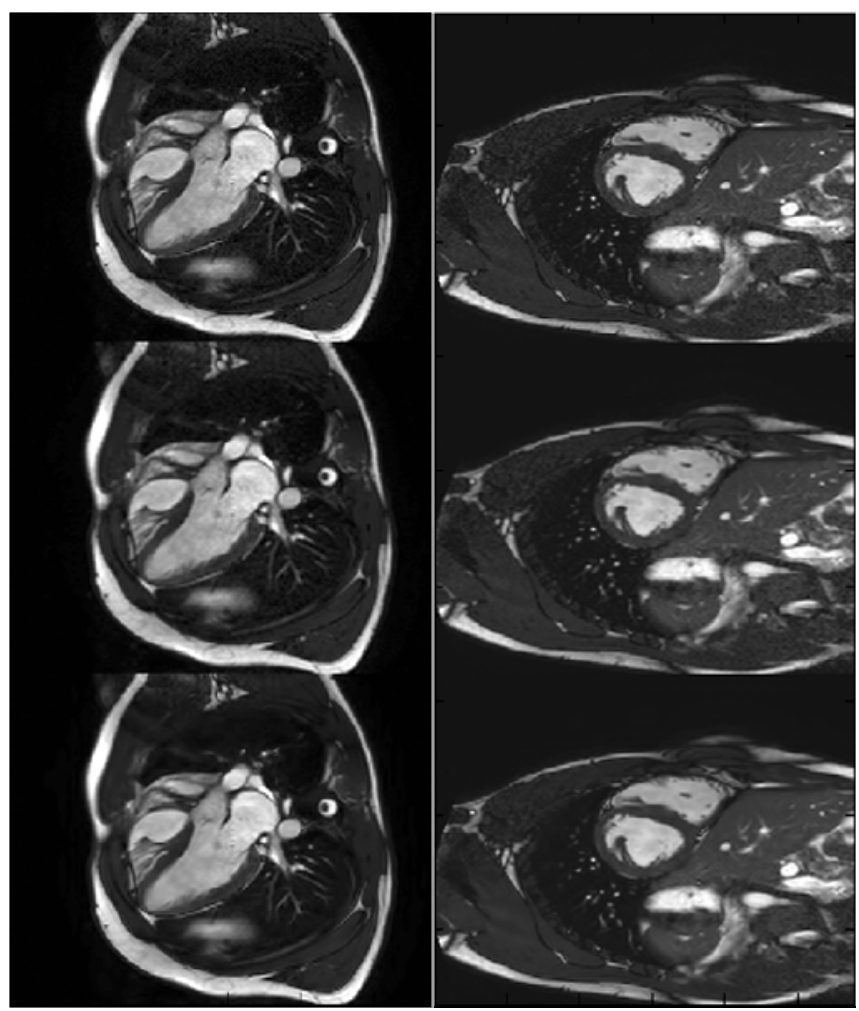

Fig.2. Images from top to bottom: low SNR cardiac images, denoised images with Wiener filter, images denoised with our method.

\section{LOW SNR MR IMAGE ENHANCEMENT}

In this section, we use our spatially adaptive algorithm to improve the visual quality of low SNR MR images.

We implement our algorithm in the discrete complex wavelet domain (DCWT) [19]. Notwithstanding, ordinary wavelet transform is an efficient computational algorithm and sparse representation that has excellent performance in many signal processing applications, yet it suffers from several fundamental shortcomings including the lack of shift invariance and poor directional selectivity [19]. In fact, although ordinary wavelet transform is optimal for a large class of 1-D signals, it only represents point-singularities efficiently and in 2-D domain it is less efficient for line-and curve-singularities (edges). Therefore, ordinary wavelet transform does not possess these optimality properties for 2D signals such as natural images, and the development of 2D multiscale transforms that isolate edges with different orientations in different subbands and represent edges more efficiently than the separable DWT, such as DCWT, has been considered. In this paper the 6-tap filter proposed in [20] is used for each dimension of DCWT.

Fig.2 shows a cardiac MR image denoised with our method and Wiener filtering in the DCWT domain with 3 levels. The Wiener filter can be obtained by using Gaussian prior distribution and modeling noise with AWGN $\left(\right.$ denoised data $=\frac{\text { variance of noise free data }}{\text { variance of noisy data }} \times$ noisy data $)$. We understand from this figure that our method is able to reduce the noise while preserving the edges and main features of MR images. Another advantage of our algorithm is its complexity. In practice, our spatially adaptive algorithm is very fast. For example, on an Intel Core Duo $1.83 \mathrm{GHz}$ personal computer with $2 \mathrm{~GB}$ RAM, the average $\mathrm{CPU}$ time in MATLAB environment for processing $512 \times 512$ images using our spatially adaptive denoising algorithm is about $2 \mathrm{~s}$.

The effect of the window size is illustrated in Fig.3. We can see that large window sizes produce blurred images.
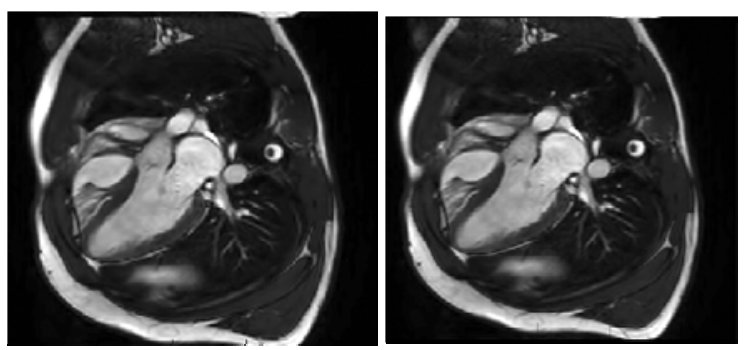

Fig.3. Left: denoised image with our method for window size $3 \times 3$. Right: denoised image with our method for window size $15 \times 15$.

\section{BD IN WAVELET DOMAIN}

In this section we complete the introduced denoising algorithm to improve the visual quality of blurred MR images by applying a BD algorithm in the wavelet domain. Although up to now a large number of $\mathrm{BD}$ algorithms have been introduced [12-16], [22-27], many of them are sensitive to noise and fail in a noisy environment [12-13] and many of the recently published methods that try to dominate this problem are time consuming [22-24]. In this paper, we use an iterative process similar to the LucyRichardson algorithm based on the maximum likelihood criterion [12-13] for BD in low-pass subband in the wavelet domain. Even though the Lucy-Richardson algorithm is well known, it has many variations which are stated to be fast and not affected by noise [14]. We use an available version 
of this algorithm in MATLAB that is a more accurate and fast version of the Lucy-Richardson algorithm. The main reason of applying $\mathrm{BD}$ on low-pass subband is that the noise mainly contaminates the high-pass subbands [8]. For example, we add an additive white Gaussian noise (with variance 20 ) to a $144 \times 144$ grayscale image and the signalto-noise ratio for corresponding subbands in 3 scales are $0.9145,-3.5094$, and -4.9150 , respectively. Note that in our method we obtain at first (approximately) noise-free data using the proposed MAP-based denoising in Section 2, and then apply BD algorithm to this data (Fig.4). The results of a sample MR image restoration with our algorithm are illustrated in Fig.5. In this figure, we also compare the results of our algorithm with the method proposed in [1214] (applying BD algorithm in the image domain). Note that since some details may be better visualized by the latter method, in some cases a combination of the results obtained by both algorithms may be beneficial. An additional application of our algorithm to a simulated blurry image can be seen in Fig.6.

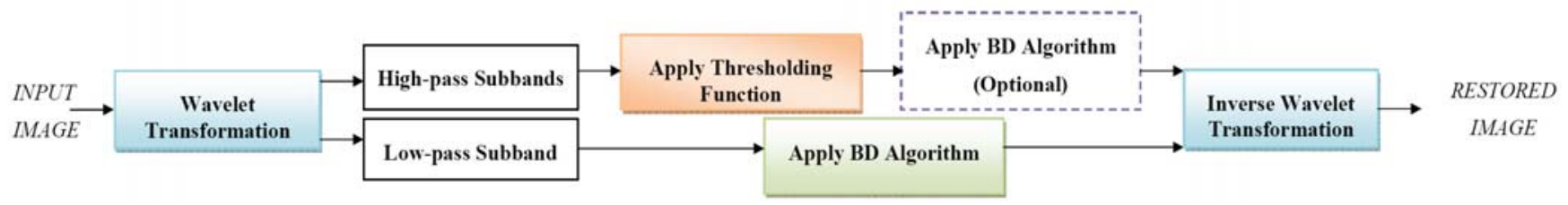

Fig.4. The block diagram of image enhancement in wavelet domain. Note that in some cases in addition to applying BD to low-pass subband, we also apply BD algorithm to other subbands (after denoising).

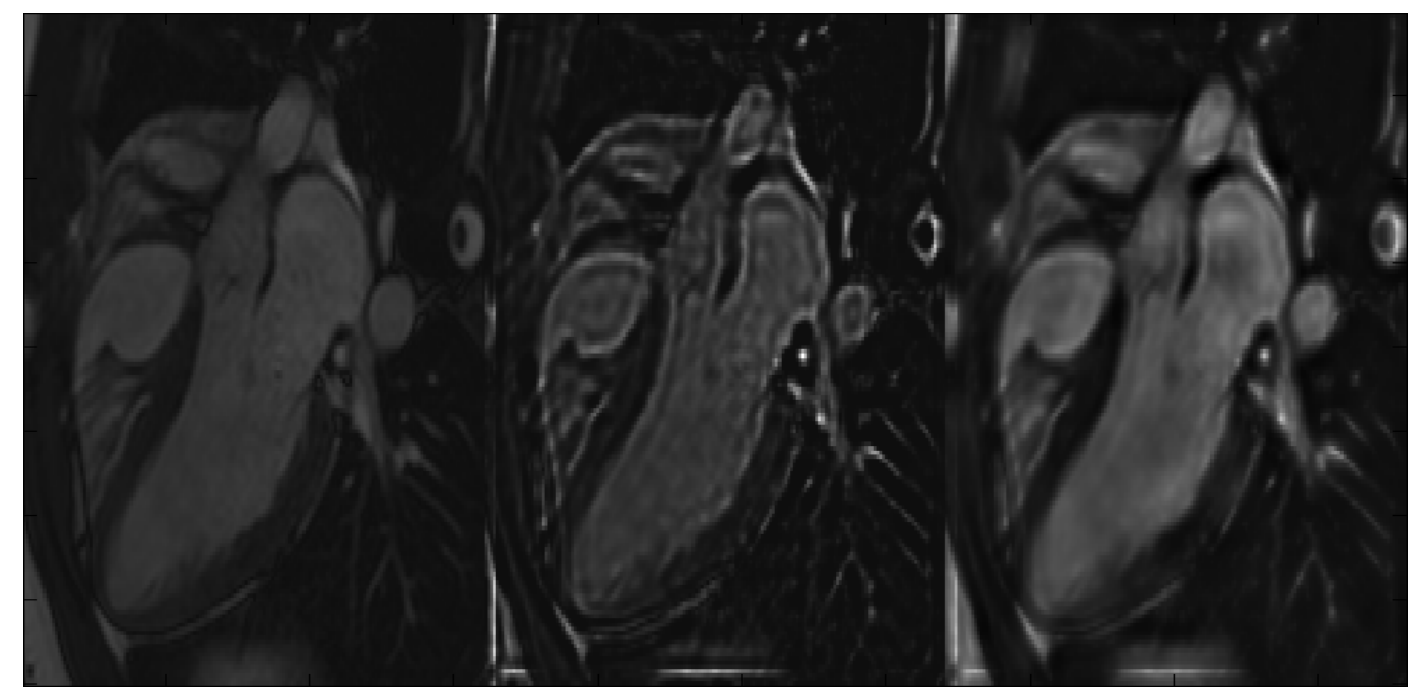

Fig.5. From left to right: initial image, restored image produced from applying BD algorithm in image domain, and image restored with our algorithm.

Table 2. Comparison between PSNRs, and MIs of themethod proposed in this paper (and other versions of this algorithm for other choices of distributions of signal and noise) with the method proposed in [12-14] for a $128 \times 128$ MR Image

\begin{tabular}{|c|c|c|c|c|c|c|c|c|}
\hline $\begin{array}{c}\text { Level } \\
\text { of } \\
\text { Noise }\end{array}$ & PSNR & MI & PSNR & MI & PSNR & MI & PSNR & MI \\
\hline & $\begin{array}{c}\text { Proposed } \\
\text { Method in } \\
{[12-14]}\end{array}$ & \multicolumn{2}{|c|}{$\begin{array}{c}\text { Our Method with } \\
\text { Laplacian Prior, } \\
\text { Gaussian Noise }\end{array}$} & \multicolumn{2}{|c|}{$\begin{array}{c}\text { Our Mith Gaussian Prior, } \\
\text { Gaussian Noise }\end{array}$} & Our Method \\
\hline$\zeta=5$ & $\mathbf{3 1 . 9 5}$ & 1.69 & 30.04 & 1.64 & 29.82 & 1.62 & 31.65 & $\mathbf{1 . 7 5}$ \\
\hline$\zeta=10$ & 26.59 & 1.39 & 26.52 & 1.50 & 26.32 & 1.48 & $\mathbf{2 7 . 1 0}$ & $\mathbf{1 . 6 0}$ \\
\hline$\zeta=15$ & 23.19 & 1.16 & 23.81 & 1.42 & 23.63 & 1.38 & $\mathbf{2 4 . 0 6}$ & $\mathbf{1 . 4 8}$ \\
\hline$\zeta=25$ & 18.74 & 0.79 & 19.69 & 1.26 & 19.65 & 1.24 & $\mathbf{1 9 . 8 5}$ & $\mathbf{1 . 3 2}$ \\
\hline$\zeta=40$ & 14.71 & 0.54 & 15.80 & 1.09 & 15.79 & 1.07 & $\mathbf{1 5 . 9 0}$ & $\mathbf{1 . 1 2}$ \\
\hline
\end{tabular}




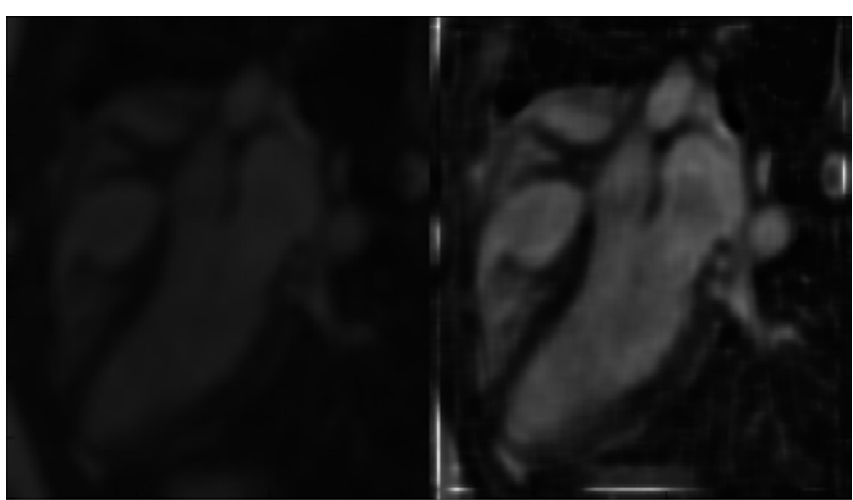

Fig.6. Left image: initial data. Right image: restored image with our algorithm.

A proper way of carrying out the performance test is to start out with a clean ground truth image, then add noise to it based on a realistic distribution from MRI, use the different algorithms to improve image quality and then compute the mean square error (or an equivalent metric, e.g., mutual information) between the ground truth image and the noisy and deblurred images. For this reason we use a $128 \times 128$ image and contaminate it with Rician noise with different levels and apply a Gaussian point spread function (PSF) on it to simulate a blurry and noisy MR image (usually PSF is modeled as a low-pass filter [28], however we also tested our algorithm for box-type, $45^{\circ}$ rotated box-type and inverse-quadratic blur functions and similar results were obtained). Then we use our algorithm for visual quality improvement and compare the results with the method proposed in [12-14] in terms of peak signal-to-noise (PSNR) ratio and mutual information (MI). The results are concluded in Table 2. Note that in this table we also show the effect of the choice of the statistical distributions for signal and noise on the performance of our algorithm. From the results it is clear that our algorithm mostly outperforms the others. In addition, we can derive that wavelet-based methods have better results than image-based methods especially for the presence of high level of noise. Note that for low level of noise, the method proposed in [12-14] has higher PSNR (but lower MI). The main reason is that the initial assumption in our method is using Rayleigh distribution for noise that is true only for high level of noise.

As we explained in Figure 4, in some cases it is better to use BD also in the high-pass subband. Figure 7 shows the effect of using the deconvolution in high-pass subband. Medical images are also characterized by another parameter - contrast to noise ratio (CNR) defined as

$$
\mathrm{CNR}=\frac{\left|\mu_{\mathrm{ROI1}}-\mu_{\mathrm{ROI} 2}\right|}{\sigma}
$$

where $\mu_{\mathrm{ROI}}$ is the mean signal value computed for a small region of interest (ROI). The noise standard deviation $\sigma$ is computed from a large region outside the object, which represents the background noise. In Fig.7, we also compare the SNRs and CNRs of original and enhanced images. For original image, SNR for ROI1 is 34.68 and for ROI2 is 36.88 and CNR is 2.2 while for the enhanced image, SNR for ROI1 is 140.99 and for ROI2 is 131.80 and CNR is 9.19 . This results show the gain in SNR without losing CNR. In this paper the $\mathrm{BD}$ is performed in the wavelet domain to avoid noise in the image domain. To show advantages of this approach we compare it with using the deconvolution algorithm in the image domain after performing the denoising. The obtained CNR for the proposed image in Fig.7 after performing soft thresholding in the discrete complex wavelet domain and then applying the LucyRichardson algorithm is 8.63 that confirm the effectiveness of our algorithm.

\section{CONCLUSION}

This paper presents a new fast denoising method in the discrete complex wavelet domain. We use maximum a posteriori estimator based on a local Laplace prior and Rayleigh noise and obtain a new spatially adaptive wavelet based denoising algorithm. We implement our algorithm for visual quality improvement of low SNR MR images and obtain denoised images while preserving the main features of clean images such as edges. For those cases in which the input image is also blurred, we complete our algorithm by applying a blind deconvolution algorithm to appropriate wavelet subbands (after denoising) and obtain the final restored data.

We can use more complicated prior distributions for clean data in the wavelet domain (e.g., see [19] for an extension to mixture prior) and better blind deconvolution algorithms to obtain better results. We can also extend this work for other applications such as super resolution reconstruction in MRI.

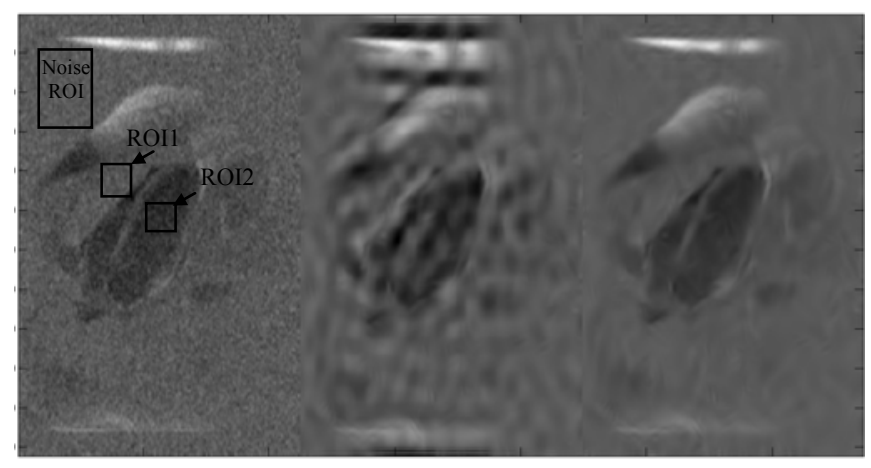

Fig.7. The effect of using BD in high-pass subband (in Figure 4) for a noisy MR image. Left image shows the initial image, the middle image shows the produced image using only BD in lowpass subband and the right image illustrates the results of using $\mathrm{BD}$ in all wavelet subbands. For left image SNR for ROI1 is 34.68 and for ROI2 is 36.88 and CNR is 2.2 while for the last image SNR for ROI1 is 140.99 and for ROI2 is 131.80 and CNR is 9.19 .

\section{REFERENCES}

[1] Vojtíšek, L., Frollo, I., Valkovič, L., Gogola, D., Juráš, V. (2011). Phased array receiving coils for low field lungs MRI: Design and optimization. Measurement Science Review, 9, 61-67.

[2] Song Huettel, A.W., McCarthy, G. (2009). Functional Magnetic Resonance Imaging, 2nd ed. Sunderland, MA: Sinauer Associates, Inc. 
[3] Donoho, D.L., Johnstone, I.M. (1994). Ideal spatial adaptation by wavelet shrinkage. Biometrika, 81, 291-294.

[4] Donoho, D.L. (1995). Denoising by soft-thresholding. IEEE Trans. on Information Theory, 41, 613-627.

[5] Mihcak, M.K., Kozintsev, I., Ramchandran, K., Moulin, P. (1999). Low complexity image denoising based on statistical modeling of wavelet coefficients. IEEE Signal Proc. Letters, 6, 300-303.

[6] Crouse, M.S., Nowak, R.D., Baraniuk, R.G. (1999). Analysis of multiresolution image denoising schemes using a generalized Gaussian and complexity priors. IEEE Trans. on Information Theory, 45, 909-919.

[7] Malfait, M., Roose, D. (1997). Wavelet-based image denoising using a markov random field a priori model. IEEE Trans. on Image Processing, 6, 549-565.

[8] Crouse, M.S., Nowak, R.D., Baraniuk, R.G. (1998). Wavelet-based statistical signal processing using hidden Markov models. IEEE Trans. on Signal Processing, 46, 886-902.

[9] Rabbani, H., Vafadust, M., Gazor, S. (2006). Image denoising based on a mixture of Laplace distributions with local parameters in complex wavelet domain. In IEEE Int. Conference on Image Processing, October 8-11, 2006. Atlanta, GA, 2597-2600.

[10] Nowak, R.D. (1999). Wavelet-based rician noise removal for magnetic resonance imaging. IEEE Trans. Image Processing, 8, 1408-1419.

[11] Chang, S.G., Yu, B., Vetterli, M. (2000). Adaptive wavelet thresholding for image denoising and compression. IEEE Trans. Image Processing, 9, 1532-1546.

[12] Richardson, W.H. (1972). Bayesian-based iterative method of image restoration. JOSA, 62 (1), 55-59.

[13] Lucy, L.B. (1974). An iterative technique for the rectification of observed distributions. Astronomical Journal, 79 (6), 745-754.

[14] Fish, D.A., Brinicombe, A.M., Pike, E.R. (1995). Blind deconvolution by means of the RichardsonLucy algorithm. J. of the Optical Society of America A, 12 (1), 58-65.

[15] Stockham, T.G., Cannon, T.M., Ingebretsen, R.B. (1975). Blind deconvolution through digital signal processing. In Proc. IEEE, 63 (4), 678-692.

[16] Cannon, M. (1976). Blind deconvolution of spatially invariant image blurs with phase. IEEE Trans. on Acoustic, Speech, and Signal Processing, 24 (1), 58-63.

[17] Rabbani, H. (2008). Statistical modeling of low SNR magnetic resonance images in wavelet domain using Laplacian prior and two-sided Rayleigh noise for visual quality improvement. In International Conference on Information Technology and Applications in Biomedicine (ITAB 2008), May 30-31, 2008. IEEE, 116-119.
[18] Rabbani, H., Vafadust, M. (2008). Image/video denoising based on a mixture of Laplace distributions with local parameters in multidimensional complex wavelet domain. Signal Processing, 88 (1), 158-173.

[19] Rabbani, H., Nezafat, R., Gazor, S. (2009). Waveletdomain medical image denoising using bivariate Laplacian mixture model. IEEE Trans. on Biomedical Engineering, 56 (12), 2826-2837.

[20] Selesnick, I.W., Kingsbury, N., Baraniuk, R.G. (2005). The dual-tree complex wavelet transforms - a coherent framework for multiscale signal and image processing. IEEE Signal Proc. Magazine, 9, 123-151.

[21] Kingsbury, N.G. (2000). A dual-tree complex wavelet transform with improved orthogonality and symmetry properties. In 2000 Int. Conference on Image Processing, Vol. 2, September 10-13, 2000. IEEE, 375-378.

[22] Geman, S., Geman, D. (1984). Stochastic relaxation, Gibbs distributions, and the Bayesian restoration of images. IEEE Trans. on Pattern Analysis and Machine Intelligence, 6 (6), 721-741.

[23] Derin, H., Elliott, H. (1987). Modeling and segmentation of noisy and textured images using Gibbs random fields. IEEE Trans. on Pattern Analysis and Machine Intelligence, 9, 39-55.

[24] Molina, R., Katsaggelos, A.K., Abad, J., Mateos, J. (1997). A Bayesian approach to blind deconvolution based on dirichlet distributions. In IEEE Int. Conference on Acoustics, Speech and Signal Processing (ICASSP-97), Vol. 4, April 21-24, 1997. IEEE, 2809-2812.

[25] Sroubek, F., Flusser, J. (2005). Multichannel blind deconvolution of spatially misaligned images. IEEE Trans. Image Processing, 14 (7), 874-883.

[26] Babacan, S., Molina, R., Katsaggelos, A. (2008). Parameter estimation in TV image restoration using variational distribution approximation. IEEE Trans. Image Processing, 17 (23), 326-339.

[27] Levin, A., Weiss, Y., Durand, F., Freeman, W.T. (2009). Understanding and evaluating blind deconvolution algorithms. In IEEE Conference on Computer Vision and Pattern Recognition (CVPR 2009), June 20-25, 2009. IEEE, 1964-1971.

[28] Greenspan, H., Oz, G., Kiryati, N., Peled, S. (2002). MRI inter-slice reconstruction using super resolution. Magnetic Resonance Imaging, 20 (5), 437-446.

Received June 14, 2011. Accepted September 9, 2011. 